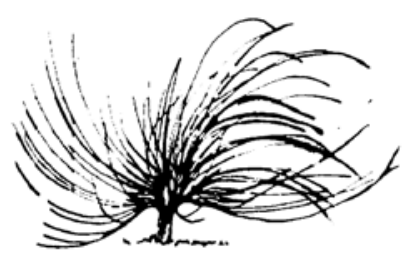

\title{
Retos de la educación: una mirada focal al papel de las nuevas tecnologías
}

\author{
María José Mora Prado \\ Universidad Nacional \\ Heredia, Costa Rica \\ atito19@hotmail.com
}

\begin{abstract}
Resumen
El presente artículo tiene como fin denotar las principales implicaciones del papel de las nuevas tecnologías en el ámbito educativo. Se abordará desde un marco general el análisis de dicho concepto, así como la intervención de estas en la educación, las exigencias que implica su incorporación, tanto para el personal docente como para el estudiantado, en qué medida se fortalece el currículo, cómo identificar los límites del uso de estas herramientas, así como evidenciar qué población es la que puede acceder a estos medios tecnológicos.
\end{abstract}

Palabras clave: currículo, docentes, estudiantes, retos, tecnologías.

\section{(C) (1) ब $\theta \Theta$}

http://dx.doi.org/10.15359/rep.12-1.6

Recibido: 13 de junio de 2016-Aprobado: 9 de mayo de 2017

1 Bachiller en la Enseñanza de los Estudios Sociales y Educación Cívica. Postulante a la Licenciatura en Pedagogía con Énfasis en Didáctica, ambas en la Universidad Nacional. 


\begin{abstract}
This article aims to highlight the main implications of the role that new technologies play in educational settings. From a general frame of reference for the analysis of this concept, the author will describe the role these technologies play in education, their implementation demands for both teachers and students, the extent to which the curriculum is strengthen with them, how to identify the limits to the use of these technologies as well as indicating which population can have access to these technological resources.
\end{abstract}

Keywords: curriculum, teachers, students, challenges, technology

\title{
Introducción
}

$\mathrm{L}$

as nuevas tecnologías, se definen como "innovaciones en microelectrónica, computación (hardware y software), telecomunicaciones y optoelectrónica - microprocesadores, semiconductores, fibra óptica - que permiten el procesamiento y acumulación de enormes cantidades de información, además de una rápida distribución de la información a través de redes de comunicación" (Cristóbal, 2009, p, 305). Existe cierta ambigüedad a la hora de definirlas como "nuevas", principalmente por el hecho de: ¿qué es lo nuevo?, ¿cómo diferenciar algo nuevo de algo viejo en la actual era digitalizada, si a diario encontramos diferentes programas, herramientas y recursos tecnológicos? Parece ser que la concepción de nuevas tecnologías nos remite a analizar las diversas herramientas digitalizadas que se han venido generando en la presente década del siglo XXI.

Es inevitable no pensar que las nuevas tecnologías forman parte de la realidad social y más aún de la educativa. Por ende, la incorporación de estas en específico, en el ámbito educativo, implica un acompañamiento de diversas estrategias y metodologías que permitan ser asertivas con la enseñanza y el aprendizaje. La generación de jóvenes de la primera y en especial la de la segunda década del S.XXI está incorporada por completo en las cuestiones tecnológicas y, en su mayoría, las dominan a cabalidad, ya que forman parte de su realidad 
mediata. En ello sobrepasan sus docentes que deben de aplicarlas en las diversas actividades de clase.

Por tal motivo, estas nuevas tecnologías exigen un compromiso claro del personal docente, en el sentido de la efectiva aplicabilidad de estas, así como del estudiantado, a la hora de hacer buen provecho de tales herramientas que tiene a su alcance. Tomando en consideración el sinnúmero de información que allí se alberga; la cual, de no ser canalizados, en este caso, hacia el fin educativo, provocará que ni estudiantes y ni docentes entiendan el fin que dichas herramientas deben tener. En este sentido, "hablar de estas herramientas, no es hablar explícitamente de mejores resultados de aprendizaje" (UNESCO, 2013, p. 29).

\section{La incorporación de las nuevas tecnologías en el ámbito educativo}

En teoría, la incorporación de las nuevas tecnologías en el ámbito educativo debería estar resguardada por un proceso de acompañamiento, principalmente del personal docente y la exigencia latente en el dominio de estas herramientas tecnológicas, que en muchos casos, son ajenas a su cotidianidad, y que, sin embargo, no por ello pueden obviarse a la hora de diseñar el currículo. Así lo expresó Lugo (como se cita en la UNESCO, 2013):

La introducción de las nuevas tecnologías en las aulas pone en evidencia la necesidad de una nueva definición de roles, especialmente, para los alumnos y docentes. Los primeros, gracias a estas nuevas herramientas, pueden adquirir mayor autonomía y responsabilidad en el proceso de aprendizaje, lo que obliga al docente a salir de su rol clásico como única fuente de conocimiento. $(\mathrm{p}, 16)$

Es necesario un replanteamiento no solo del papel que cumplen los principales agentes del proceso educativo: estudiantes y docentes, sino también, el de los contenidos, ya no basta con enseñar con base en los modelos del siglo pasado; la nueva era exige cambios constantes. Los primeros, desde la apertura de información y la capacidad consultiva que puedan tener, lo cual demandará, para el personal docente, salir de su "estado del confort". Esto no es tarea sencilla, tomando en consideración la implementación de las prácticas tradicionalistas que predominan en los centros educativos y que los mismos grupos docentes 
han acogido con conformidad. De igual forma, es trascendental analizar desde qué óptica se están utilizando estas herramientas en los salones de clase, si desde la visión de actualización tecnológica de acuerdo con el contexto en el que nos encontramos o desde una visión más pedagógica y de enriquecimiento de los procesos de enseñanza y aprendizaje.

\section{Fortalecimiento del currículo con la incorporación de las nuevas tecnologías}

La incorporación de las nuevas tecnologías de la información y la comunicación, como tal, suponen un cambio o una mejora en los programas de estudio, gracias a que abren la posibilidad de trabajar ya no solo desde el aula y con las diversas herramientas que se pueden trabajar en ella, "sino desde la comodidad de la casa e inclusive, permiten trabajar con instituciones de otros países" (Carnoy, 2004, p. 3). Por ello, se apuesta, cada vez con mayor frecuencia, a la inversión de estas en los diferentes centros educativos, como un impulso para mejorar la educación. Pero, ¿en qué medida se fortalece el currículo con la intervención tecnológica?

Como primer aspecto, es ineludible no pensar que las nuevas tecnologías abren paso a una expansión del currículo en todos los sentidos, desde la cantidad de información a la cual se puede acceder, así como a las diversas actividades didácticas que se pueden llevar a cabo a partir de estas, es decir, son una herramienta activa que enriquece el currículo establecido. Según lo desarrollado por la UNESCO (2013), la incorporación de estas "ayuda a los estudiantes a aprender juntos, ya que permite el trabajo por medio de diversas herramientas, tales como: redes sociales, trabajo colaborativo, producciones colectivas. Así, el uso de las tecnologías en la educación no implicarían únicamente el intercambio y la interacción, sino que contribuyen a visibilizar y valorar la diversidad de cultura desde el enfoque de los derechos humanos" (p. 26).

En segundo lugar, estas interfieren en la motivación del estudiantado a la hora de aprender los contenidos de clase, trabajar con herramientas tecnológicas hará aún más atractivo el proceso de enseñanza y aprendizaje. Es importante tomar en consideración que será un proceso más atractivo, si el docente cuenta con las capacidades para crear material con base tecnológica, que sea significativo para el estudiantado; de lo contrario, no será más que una clase como la habitual. Asimismo, como lo expresó Fernández, el personal docente puede ser 
un muy buen comunicador, pero si no tiene la motivación del grupo, será muy difícil que logre conseguir los objetivos deseados (p, 3). Es menester que el personal docente tenga claro con qué tipo de herramientas tecnológicas está trabajando (dominio): no se puede permitir improvisar dentro del aula.

En tercer lugar, la incorporación de estas herramientas tecnológicas abre paso a un mundo infinito de información. Claro está, es preciso identificar los límites que deben existir en cuanto al uso, o mejor dicho, al buen uso de esta amplitud informacional. Como lo detalló Cabero (2010): "el docente cuenta como nunca con una 'galaxia tecnológica' que se presenta en diferentes sistemas simbólicos, y que gracias a la digitalización y convergencia de estas, brinda a los educadores, un gran cúmulo de posibilidades para su explotación en el terreno educativo" (p. 40).

En cuarto lugar, se podría destacar la importancia del aprender a hacer desde las nuevas tecnologías. Se debe entender estas herramientas desde el enfoque de aporte, es decir, que vengan a enriquecer el currículo educativo y no como válvula de escape para las obligaciones que al personal docente le competen. De manera puntual, con la simple incorporación de herramientas como Movie Maker y donde, con base en la contextualización del estudiantado, este salga a plasmar esa realidad por medio de un vídeo, le dará un valor significativo a dicha herramienta: siempre es importante partir de lo más cercano y tangible de la realidad a la cual el grupo de estudiantes está expuesto. Tal y como lo destacó la UNESCO (2013), "estas nuevas tecnologías contribuyen en la construcción de soluciones o resolución de problemas, así como el desarrollo de distintos tipos de producciones, por ejemplo: creaciones audiovisuales, lo cual aporta considerablemente al desarrollo de la creatividad en los estudiantes" (p. 26).

Por otra parte, como aspecto número cinco, se podría decir que se logra la interactividad con mayor fuerza que desde la no utilización de estas nuevas herramientas tecnológicas. Este aspecto es de vital importancia y al cual se le debe prestar especial atención, ya que la interactividad, de ser aplicada de forma asertiva, donde se potencie el desarrollo de ideas propias en el estudiantado, dará paso, sin lugar a dudas, a su desenvolvimiento ante las demás personas y, sobretodo, fomentará el pensar crítico; fundamental para el desarrollo de pensamiento e ideas propias, así también, como la potenciación de la expresión oral. Según lo desarrollado por Fernández (s. f), "el alumno 
puede interactuar, se puede comunicar, puede intercambiar experiencias con otros compañeros del aula, e inclusive con otros centros educativos, enriqueciendo grandemente el aprendizaje" (p. 4).

Estas son algunas de las consideraciones que se deben tomar en cuenta a la hora de calificar las nuevas tecnologías como un medio para el fortaleciendo del currículo. Es importante aplicarlas desde la óptica del cuestionamiento, en este caso, basadas principalmente en el qué, el cómo y el para qué de su implementación.

\section{¿Uso o abuso de las nuevas tecnologías en el aula?}

Como se evidenció, la incorporación de estas aporta aspectos positivos y específicos al ámbito educativo, sin embargo, la exposición a todas estas herramientas, puede generar adicciones muy severas en los grupos de jóvenes, por lo cual, debe existir un uso racional, que permita el aprendizaje desde el enfoque tecnológico, pero que dé espacio para la potencialización de otras actividades, no específicamente relacionadas con tecnología (actividades motoras, visuales, auditivas). Esto implica un compromiso claro por parte de todo el sistema educativo: estudiantes, docentes, padres y madres de familia, en general. No se puede justificar la no aplicación de estas en el aula, así como tampoco su implementación excesiva; debe existir un límite, que permita no caer en ambos extremos. En este sentido, Echeburúa, y Corral (2010), expresan lo siguiente:

El uso de las nuevas tecnologías, impone a los adolescentes y adultos una responsabilidad de doble dirección; los jóvenes pueden enseñar a sus padres en el uso de estas nuevas tecnológicas, su lenguaje y las posibilidades de crear a partir de estas y los padres deben de enseñar a los jóvenes a utilizarlas en su justa medida. Así como también intervienen los docentes, los cuales deben ayudar a los jóvenes a desarrollar la habilidad de la comunicación cara a cara. (p. 5)

Es indispensable esta relación de comunicación que debe existir entre escuela, estudiantes y familias. No se pretende aquí sancionar únicamente la adicción de jóvenes hacia las nuevas tecnologías, sino en general a estos tres agentes interventores del proceso educativo. Es 
importante tomar en consideración la idea de no caer en los extremos antes mencionados y, a la hora de diseñar el currículo se debe tener presente que no todo debe ser tecnología; si bien la dominan a cabalidad, crear actividades que potencien el desarrollo de sus competencias o habilidades, por ejemplo, el cara a cara es fundamental. La juventud se ha venido acostumbrando a la virtualidad de una forma tal que han dejado de lado la expresión oral y no saben, en la mayoría de los casos, exponer ideas de forma clara y fluida. Esto se considera un problema grave dentro del abuso que puede existir, así como la mala escritura que se puede hacer visible en los mensajes de texto.

Ramón (2010, citado por Echeburúa y Corral, 2010, p. 5) expresó que deben existir ciertas bases a la hora de tomar en consideración el manejo de los recursos tecnológicos en jóvenes de una forma concientizada, los define en seis aspectos específicos:

a. Limitar el uso de aparatos y pactar las horas de uso del ordenador.

b. Fomentar la relación con otras personas.

c. Potenciar aficiones tales como la lectura, el cine y otras actividades culturales.

d. Estimular el deporte y las actividades en equipo.

e. Desarrollar actividades grupales, como las vinculadas al voluntariado.

f. Estimular la comunicación y el diálogo en la propia familia.

Dichas cuestiones son fundamentales para el desarrollo de toda persona, por lo cual no se deben dejar de lado a la hora de implementar las nuevas tecnologías en el aula; ello implica un compromiso serio con el estudiantado y el desarrollo potencial de sus habilidades como personas y entes sociales. Acá, los diversos programas de estudio deben analizar el equilibrio de ambos aspectos, así como tener presente que estos programas deben atender las necesidades de la mayoría de la población estudiantil, en este caso, no todos tienen el mismo acceso a dichas herramientas tecnológicas.

\section{¿Quién tiene acceso a las nuevas tecnologías?}

Como se planteó con anterioridad y como se ha venido desarrollando en el escrito, las nuevas tecnologías han significado un 
cambio en el currículo, sin embargo, es importante cuestionarse sobre qué población tiene acceso a estas nuevas tecnologías, ya que a pesar de los esfuerzos por la incorporación de estas en los programas de estudio recientes, existe una clara deficiencia en cuanto al alcance que, en el caso costarricense, tienen estas tecnologías en las poblaciones más lejanas del país, específicamente en las poblaciones indígenas. Estas no solo deben estar disponibles, sino ser accesibles, y para esto, la UNESCO (2013) planteó tres cuestiones específicas que deben vincularse: "la accesibilidad física, la accesibilidad curricular y la accesibilidad económica" (p. 28).

Hablar de las tecnologías va más allá de su simple incorporación en el currículo como tal, y no es únicamente un asunto de alcance por medio de la eliminación de las distancias físicas, sino que se debe hacer referencia al alcance de estas en relación con los tres puntos antes expuestos. Por citar un ejemplo concreto, a continuación se presenta la tabla comparativa (tabla 1), con el fin de evidenciar el alcance y uso que tienen las nuevas tecnologías en diferentes centros educativos indígenas de Costa Rica, para el año 2010:

Tabla 1: Índice de situación educativa, según etnia, 2010

\begin{tabular}{lrrrrrrrr}
\hline \multicolumn{1}{c}{ Etnia } & $\begin{array}{c}\text { Número } \\
\text { de centros }\end{array}$ & ISE Proceso & $\begin{array}{c}\text { Usoy } \\
\text { acceso } \\
\text { TIC's }\end{array}$ & $\begin{array}{l}\text { Titulación } \\
\text { docente }\end{array}$ & Infraestructura & Logro \\
\hline Guaymi (Ngobe) & 27 & 48,7 & 90,6 & 0,2 & 23,8 & 36,7 & 92,0 \\
\hline Cabécar & 116 & 49,9 & 89,2 & 3,1 & 27,3 & 42,0 & 87,8 \\
\hline Bribri & 69 & 56,5 & 92,4 & 8,2 & 43,1 & 43,7 & 95,1 \\
\hline Téraba(Teribe) & 9 & 60,7 & 95,4 & 0,0 & 68,4 & 39,8 & 100,0 \\
\hline Boruca(Brunka) & 25 & 63,8 & 94,5 & 0,0 & 74,4 & 49,9 & 100,0 \\
\hline Guatuso(Malekus) & 6 & 64,1 & 96,7 & 9,0 & 40,0 & 75,0 & 100,0 \\
\hline Huetares & 5 & 73,2 & 97,9 & 39,4 & 58,8 & 70,0 & 100,0 \\
\hline Chorotega & 2 & 75,1 & 92,9 & 25,1 & 87,5 & 88,9 & 81,0 \\
\hline
\end{tabular}

Estado de la Educación con datos del Departamento de Análisis Estadístico del MEP y el Departamento de Educación Indígena del MEP. (Borge, 2012, p. 18).

Tomando como referencia únicamente el apartado sobre el uso y el acceso de las nuevas tecnologías, que es lo que nos interesa analizar, en las diferentes regiones indígenas del país, quedó en evidencia, que el alcance es visiblemente limitado, inclusive con índices de no aplicabilidad de estas en el currículo, lo cual denota que, si bien, en las regiones 
más mediatas o cercanas a focos centrales se invierte gran cantidad de recursos en la incorporación de tecnología, las regiones lejanas se están quedando por fuera de dicho proceso. Es de suponer que un cambio en el currículo implica el esfuerzo por un avance global, no simplemente "maquillar" y dar mantenimiento a las instituciones más cercanas y de fácil acceso. Este debe ser un esfuerzo que debe hacerse visible desde los cuerpos docentes y su compromiso social como tal; no se puede esperar que sean los entes centrales los que tomen la iniciativa del cambio.

\section{Conclusiones}

Es importante el cuestionamiento constante que, desde la educación, se debe hacer sobre la implementación de las herramientas tecnológicas dentro del aula. Estas no deben verse como la única opción en la educación, así como tampoco se debe justificar la no aplicación de estas en las diversas estrategias didácticas que se vayan a implementar. Existen aspectos positivos, como los expuestos, que denotan a las nuevas tecnologías como una herramienta apta para potenciar y facilitar el proceso de enseñanza y aprendizaje. Asimismo, es sabido que se deben tener limitaciones y reglas específicas en cuanto a su implementación, estas deben tener un objetivo claro y alcanzable, así como también hacer hincapié en la realidad presente.

Por otra parte, no se debe generar la estricta aplicación de estas en todas las estrategias didácticas de aula, se debe potenciar el desarrollo de cualidades personales, por medio del dialogo directo, el trabajo en equipo y demás aspectos que se consideran necesarios para el desenvolvimiento personal. Por tanto, hablar de incorporación tecnológica no es hablar de un aprendizaje en definitiva, es importante que nos cuestionemos sobre la efectiva aplicabilidad y los objetivos que esperamos lograr con su implementación.

Es a partir de estos cuestionamientos que nos enfrentamos ante una educación que, muy distante en comparación a la desarrollada en el siglo pasado, exige, entre otras cosas, la actualización constante por parte de docentes, esto si tomamos en consideración lo "nuevo" y lo que implica. Por otra parte, la incorporación tecnológica en el ámbito educativo evidencia un constante confrontamiento entre lo que el profesorado ofrece en el salón de clase, o lo que puedo encontrar en sus dispositivos móviles el estudiantado, tal como lo puede ser un juego. 
Por ende, la parte activa y creativa del personal docente en el espacio áulico, que le permita estar a la vanguardia de los recursos tecnológicos que existen actualmente, será un factor clave en los procesos motivacionales del estudiantado, así como también en el proceso de enseñanza y aprendizaje. En este sentido, debemos cuestionarnos más en profundidad, ¿cuán preparados se encuentran los cuerpos docentes para hacer frente a la implementación tecnológica?

\section{Referencias}

Borge, C. (2012). Costa Rica: Estado de la educación en territorios indigenas. Cuarto informe del Estado de la Educación. Recuperado de http://www.estadonacion.or.cr/files/biblioteca_virtual/ educacion/004/borge_educacion_indigena.pdf

Cabero, J. (2010). Los retos de la integración de las TIC en los procesos educativos. Límites y posibilidades. Revista perspectiva educacional, 49(1), p. 32-61. Recuperado de https://dialnet.unirioja.es/ descarga/articulo/3579891.pdf

Carnoy, M. (2004). Las TIC en la enseñanza: Posibilidades y retos. FUOC. Recuperado de http://www.uoc.edu/inaugural04/esp/carnoy 1004.pdf

Cristóbal, J. (2009). El concepto de tecnología de la información. Benchmarking sobre las definiciones de las TIC en la sociedad del conocimiento. ZER, 14(27), p. 295-318. Recuperado de http:// www.ehu.eus/zer/hemeroteca/pdfs/zer27-14-cobo.pdf

Echeburúa, E. y Corral, P. A. (2010). Adicción a las nuevas tecnologías y a las redes sociales en jóvenes: Un reto. Revista digital Adicciones, 22(2). Recuperado de http://www.redalyc.org/ pdf/2891/289122889001.pdf

Fernández, I. (s. f). Las TIC en el ámbito educativo. DNI: 48937600-V. Recuperado de: http://www.eduinnova.es/abril2010/tic_educativo.pdf

UNESCO, Severin, E. (colaborador). (2013). Enfoques estratégicos sobre las TIC en educación en América Latina y el Caribe. (Chile: Oficina Regional de Educación para América Latina y el Caribe). Recuperado de http://www.unesco.org/new/fileadmin/MULTIMEDIA/FIELD/Santiago/images/ticsesp.pdf 